
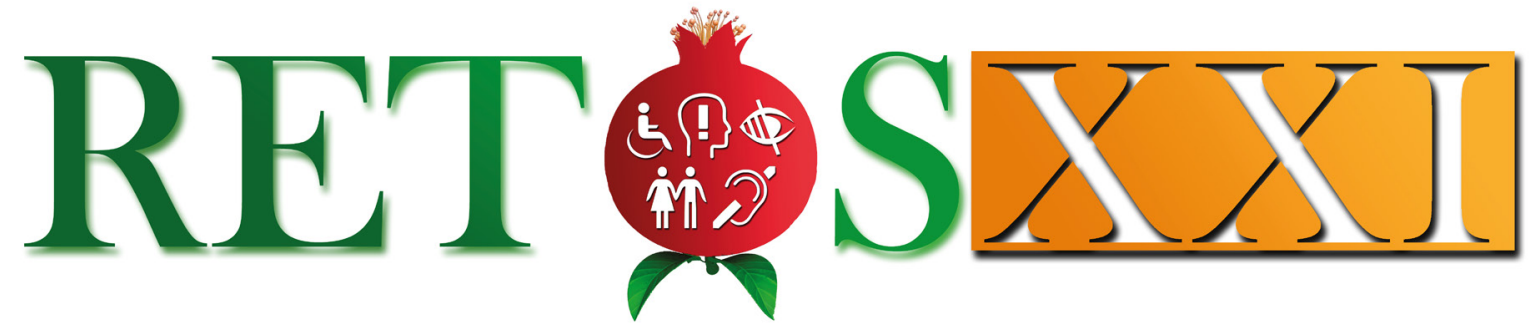

LA NECESIDAD DE INVESTIGACIÓN-ACCIÓN COMO MEJORA EDUCATIVA THE NEED FOR RESEARCH-ACTION AS AN EDUCATIONAL IMPROVEMENT

Reseña de la Tesis Doctoral “Desarrollo y validación de una aplicación Web y CD educativa inclusiva para el aprendizaje de la Lengua de Señas Panameña"

Vladimir Villarreal

Volumen 3, 2019.

Enviado: $23 / 07 / 2019$

Aceptado: 30/07/2019 


\title{
RESEÑA DE LA TESIS DOCTORAL “DESARROLLO Y VALIDACIÓN DE UNA APLICACIÓN WEB Y CD EDUCATIVA INCLUSIVA PARA EL APRENDIZAJE DE LA LENGUA DE SEÑAS PANAMEÑA"
}

\author{
Vladimir Villarreal 1
}


Como miembro del tribunal de evaluación de la tesis realizada por la reciente doctora Lineth Alaín, profesora de la Universidad Tecnológica de Panamá, y defendida el día 21 de junio de 2019 en la Universidad de Granada (España), quiero hacer una breve descripción del producto de la tesis y del proceso de su evaluación en el que participé directamente. Empezando por el final, la comisión estuvo conformada por dos profesores de la Universidad de Granada, que actuaron en calidad de Presidente y Secretario de la misma. Como presidente actuó el Dr. Sola Martínez, catedrático de Educación Especial y Director del Grupo de Investigación área de la misma Universidad. La secretaría de la comisión recayó sobre el Dr. García Guzmán, decano de la Facultad de Educación, Tecnología y Economía y especialista en atención a la diversidad del alumnado. La Universidad Nacional de Educación a Distancia (UNED) estuvo representada por la Dra. Sevillano García, catedrática de Tecnología Educativa, y la Universidad de Córdoba por la Dra. Marín Díaz, profesora especialista en tecnología educativa. Y por parte de la Universidad Tecnológica de Panamá tuve el honor de participar, al resultar seleccionado por la Escuela de Postgrado de la Universidad de Granada.

La génesis de la tesis doctoral, que culminó con la presentación y validación de la aplicación EnSenias para enseñar y aprender la Lengua de Señas Panameña (LSP), se encuentra en la Convención Interamericana para la eliminación de todas las formas de discriminación contra las personas con discapacidad, de la Organización de los Estados Americanos (OEA, 1999) y en el reconocimiento de la Lengua de Señas por parte de la República de Panamá en su la ley 1 del 28 de enero de 1992, por la cual se protege a las personas con discapacidad auditiva $y$ se reconoce la lengua de señas panameñas como lengua natural de las personas sordas (SENADIS, 2012).
La finalidad de este trabajo es trasladar ese potencial de ventajas e inclusión, ese avance y mejora en la calidad de vida, esa alternativa y apoyo al desconocimiento generalizado social de la LSP, al contexto panameño. Su objetivo fue crear y validar una aplicación Web y CD con el vocabulario inicial perteneciente a la LSP reconocida oficialmente como recurso educativo para su aprendizaje por parte de cualquier usuario interesado, accesible para todas las personas, pues todas pueden ser usuarios potenciales de la LSP. Para personas sin discapacidades, pero también para personas con discapacidades, trastornos y desigualdades variadas.

El informe de la tesis evaluada sigue la estructuración de informes y memorias de investigación. Siguiendo una ordenación desde la introducción donde se hace referencia a la problemática y la relevancia de la misma desde el contexto de discapacidad auditiva, hasta la aplicación Web que se realiza y las conclusiones. Desarrollándose en total siete capítulos, y las referencias bibliográficas. Un primer apartado contiene la descripción del escenario y estado del arte de la dimensión trabajada. Dentro de esta fundamentación teórica y revisión de obras afines se presentan tres capítulos. El primero, titulado "discapacidad auditiva y lengua de señas panameña", se abordan los aspectos relativos a la audición y especialmente a su disminución o pérdida, haciendo especial énfasis en pérdidas graves o totales de audición; y sus consecuencias para la comunicación, y más especial aún en la lengua alternativa a la oral cual es la LS y en el contexto panameño la LSP. El segundo presenta la necesaria "educación del alumnado con pérdidas de audición en el contexto panameño", abordando la filosofía y la realidad de la educación inclusiva en el escenario de la escuela para todos como la mejor de las 
opciones posibles para la educación formal del alumnado con discapacidad auditiva.

Se culmina esta revisión teórica sobre los precedentes del trabajo presentado con el capítulo tercero, "Tecnología de la Información y la Comunicación (TIC) como apoyo a la discapacidad auditiva", el cual presenta información sobre las TIC como herramientas que apoyan la enseñanza y aprendizaje, y se proporciona una revisión desde el escenario del contexto nacional e internacional del aporte de las TIC, en el desarrollo de aplicaciones, recursos y programas para apoyar la comunicación y aprendizaje de las LS, especialmente las afines a la LSP.

El capítulo cuarto de la tesis adquiere la importancia por su carácter metodológico investigador. Titulado "método de investigación para el diseño y validación de plataforma EnSenias", supone el esqueleto y algoritmo metodológico seguido en la investigación, se esboza los problemas identificados para esta investigación y los objetivos y acciones correspondientes de desarrollo, así como la naturaleza de la acción investigativa y el método investigador. Respondiendo al modelo de Investigación Basada en el Diseño (IBD), que supone una evolución y concreción del modelo de Investigación-Acción (I-A), generado por Lewin en 1946 y desarrollado en el terreno de la investigación educativa entre otros por Carr y Kemmis (1986), Stenhouse $(1985,1991)$ y Anderson $(1999,2000)$. La IBD parte desde el desarrollo de una aplicación Web para dar solución a un problema identificado y su validación en tal escenario. El paradigma de IBD proviene de la ingeniería y la programación, así como de otras ciencias aplicadas (De Benito y Salinas, 2016). Para el desarrollo de la aplicación Web se emplea la metodología de desarrollo de ingeniería de software de Pressman (2002), la cual orienta el desarrollo de la aplicación, dando como resultado la aplicación Web EnSenias.

En el siguiente apartado dedicado a los resultados y vertebrado en dos capítulos se presenta la aplicación EnSenias, y su correspondiente validación. Detallándose de esta forma en el capítulo cinco el correspondiente proceso de desarrollo y optimización de esta aplicación, describiendo pormenorizadamente, en primer lugar, cada una de las fases realizada de la misma, con sus correspondientes actividades, previas a la descripción de la plataforma, producto final en su versión final denominada EnSenias. Detallándose la construcción de 1253 palabras-queremas en LSP, los cuales se representan por medio de diversos objetos: vídeo, audiodescripción, descripción textual, categorías gramaticales, imágenes, etc., los cuales ofrecen el potencial inclusivo de la misma a una diversidad de personas y capacidades.

En el capítulo es el seis, sobre "validación de la plataforma EnSenias por la población usuaria”, se presentan los resultados correspondientes a la validación de la aplicación con los usuarios potencialmente de la misma: estudiantes sordos, asociaciones de sordos, intérpretes de LSP, docentes especialistas y generalistas de distintos niveles, progenitores, etc. Se realiza también la validación en el contexto internacional participando docentes universitarios expertos en discapacidad sensorial de la universidad Marta Abreu de las Villa (Santa Clara) y representantes de la Asociación de sordos de Cuba, profesionales personas sordas del Instituto Nacional de Sordos de Colombia (INSOR) y docentes de la Universidad Pedagógica Nacional de Colombia. 
Se parte de un análisis cualitativo basado en el análisis de contenido de información declarada por los usuarios y recogida mediante la técnica de focus groups, al que se le añaden análisis descriptivos de cada uno de los grupos (medias y desviaciones típicas) y diferenciales ( $U$ de Mann Whitney y, en su caso, HSD de Tukey). Presentándose así los análisis descriptivos globales con la totalidad de los participantes (medias y desviaciones típicas) y correlaciónales (coeficiente de correlación de Spearman), con la finalidad de ofrecer una visión de conjunto de sus declaraciones, en forma de complemento al análisis detallado de contenido, en un marco de complementariedad de método de investigación cuantitativos y cualitativos, o métodos mixtos de investigación.

Los resultados reportan la consideración de EnSenias como un recurso necesario y prometedor para la enseñanza y aprendizaje de la LSP, de acuerdo a las expresiones, intenciones y opiniones de los distintos grupos de usuarios potenciales, mayoritariamente favorables, y las sugerencias realizadas, todas tomadas en cuenta e incorporadas en la versión final del producto.

En la última etapa de la memoria, como es de recibo, se dedica a presentar las "conclusiones, proyecciones y prospectiva de la investigación". Por un lado, un panorama global de lo conseguido y culminación de la investigación, con la finalidad de dar respuesta directa y específica a los objetivos que guiaron el proceso investigador. Los resultados contenidos en detalle en los capítulos precedentes a este, se completa el capítulo con la propuesta sobre posibilidades de aplicaciones pedagógicas del producto elaborado, una vez validado, y se establecen nuevos horizontes posibles y tópicos afines de continuación de la investigación, que perpetúen y avancen el loable objetivo de mejorar la comunicación de personas sordas en la sociedad panameña, a través de la expansión (aprendizaje) de su lengua natural: LSP, puesto que aunque sea la lengua natural de personas sordas no es exclusiva para ellos y se hace necesario que puede ser aprehendida y usada por otras personas y no solo en los contextos educativos, sino también sociales, buscando así a contribuir con una sociedad inclusiva. 


\section{REFERENCIAS}

Anderson, G. L. y Herr, K. (1999). The new paradigm wars. Is there room for rigorous practitioner knowledge in schools and universities? Educational Researcher, 28 (5), 12-21. Doi: https://doi. org/10.3102\%2F0013189X028005012

Anderson, G. L. y Jones, F. (2000). Knowledge generation in educational administration from the inside-out: The promise and perils of site-based, administrator research. Educational Administration Quarterly, 36 (3), 428-464. Doi: https://doi. org/10.1177\%2F00131610021969056

Carr, W. y Kemmis, S. (1986). Teoría crítica de la enseñanza. La investigación-acción en la formación del profesorado. Barcelona: Martínez Roca.

De Benito, B. y Salinas, J.M. (2016). La investigación basada en diseño en Tecnología Educativa. RIITE. Revista Interuniversitaria de Investigación en Tecnología Educativa, 0, 44-59. Doi: http://dx.doi.org/10.6018/ riite/2016/260631

Lewin, K. (1946). Action research and minority problems. Journal of Social Issues, 2 (4), 34-46. Recuperado de http://www.cscd.
osaka-u.ac.jp/user/rosaldo/K_Lewin_Action_ research_minority_1946.pdf

Organización de los Estados Americano (1999). Convención interamericana para la eliminación de todas formas de discriminación contra personas con Discapacidad. Recuperado de http://apw.cancilleria.gov. co/tratados/AdjuntosTratados/064dd_OEA1999\%20DISCAPACIDAD.PDF

Pressman, Roger (2002). Ingeniería del Software: Un Enfoque Práctico (5a. ed.). México MCGRAW-HILL/ Interamericana de España, S. A. U.

Secretaria Nacional de Discapacidad de Panamá (2012). Ley $N^{\circ}$ 23. Recuperado de http://www.senadis.gob.pa/wp-content/ uploads/2012/10/Ley-23.pdf

Stenhouse, L. (1985). La investigación como base de la enseñanza. Madrid: Morata, 1987.

Stenhouse, L. (1991). Investigación y desarrollo del currículo. Madrid: Morata. 